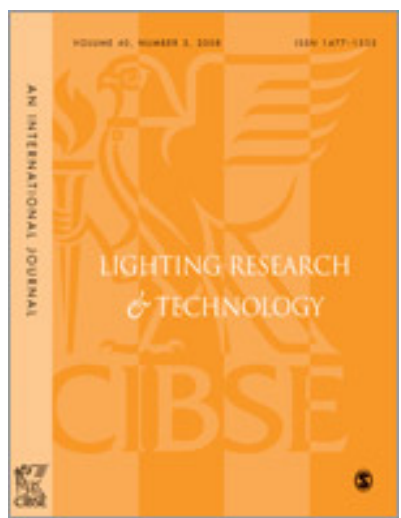

\title{
Compact illumination system with variable beam direction and beam divergence
}

\begin{tabular}{|c|c|}
\hline Journal: & Lighting Research \& Technology \\
\hline Manuscript ID & LRT-20-0096 \\
\hline Manuscript Type: & Original Manuscript \\
\hline $\begin{array}{r}\text { Date Submitted by the } \\
\text { Author: }\end{array}$ & 17-Jun-2020 \\
\hline Complete List of Authors: & $\begin{array}{l}\text { Rondelez, Nick; KU Leuven Science Engineering and Technology Group, } \\
\text { ESAT } \\
\text { Ryckaert, Wouter; KU Leuven Science Engineering and Technology } \\
\text { Group, ESAT } \\
\text { Meuret, Youri; KU Leuven Science Engineering and Technology Group, } \\
\text { ESAT }\end{array}$ \\
\hline Keywords: & Tuneable lighting, Variable radiation pattern, Lighting design \\
\hline Abstract: & $\begin{array}{l}\text { Countless approaches to optimise lighting conditions for indoor and } \\
\text { outdoor applications are described in the literature by adaptive dimming } \\
\text { schemes or control algorithms. Those methods rely on adapting the } \\
\text { emitted flux of individual luminaires in order to adjust lighting conditions } \\
\text { to varying circumstances. Complex illumination requirements are tackled } \\
\text { by controlling the output of every independent luminaire. In this case, } \\
\text { the innovation is rather in software than in hardware, and such } \\
\text { approaches are limited by how the LED fixtures can be manipulated. } \\
\text { Lighting fixtures with a tuneable radiation pattern are rare, or these } \\
\text { fixtures are rather large. This paper introduces a compact lighting } \\
\text { system with a collimated light beam of which the beam direction and } \\
\text { beam divergence can be adjusted electronically. To realise this optical } \\
\text { functionality, a focus tuneable lens and rotatable mirror are combined } \\
\text { with two custom-made aplanatic lenses in front of a compact, high- } \\
\text { brightness LED. This paper elaborates the optical design of this novel } \\
\text { illumination system and discusses the experimental performance of the } \\
\text { realised demonstrator. The system functionality is validated by } \\
\text { comparing the experimental lighting performance to optical simulations } \\
\text { conducted with ray-tracing software. With the proposed system, a fast } \\
\text { and flexible adaptation of the generated illumination distribution is } \\
\text { possible. }\end{array}$ \\
\hline
\end{tabular}


Note: The following files were submitted by the author for peer review, but cannot be converted to PDF You must view these files (e.g. movies) online.

Demonstrator.MP4

\section{SCHOLARONE Manuscripts}




\title{
Compact illumination system with variable beam direction and beam
}

\section{divergence}

\author{
N Rondelez ${ }^{a}$, WR Ryckaert Prof ${ }^{a}$, and Y Meuret Prof ${ }^{a}$ \\ a Department of Electrical Engineering (ESAT), Light and Lighting Laboratory, KU Leuven, Ghent, \\ Belgium \\ Short title: Variable light beam
}

Received 17 June 2020

Countless approaches to optimise lighting conditions for indoor and outdoor applications are described in the literature by adaptive dimming schemes or control algorithms. Those methods rely on adapting the emitted flux of individual luminaires in order to adjust lighting conditions to varying circumstances. Complex illumination requirements are tackled by controlling the output of every independent luminaire. In this case, the innovation is rather in software than in hardware, and such approaches are limited by how the LED fixtures can be manipulated. Lighting fixtures with a tuneable radiation pattern are rare, or these fixtures are rather large. This paper introduces a compact lighting system with a collimated light beam of which the beam direction and beam divergence can be adjusted electronically. To realise this optical functionality, a focus tuneable lens and rotatable mirror are combined with two custom-made aplanatic lenses in front of a compact, high-brightness LED. This paper elaborates the optical design of this novel illumination system and discusses the experimental performance of the realised demonstrator. The system functionality is validated by comparing the experimental lighting performance to optical simulations conducted with ray-tracing software. With the proposed system, a fast and flexible adaptation of the generated illumination distribution is possible.

Address for correspondence: Nick Rondelez, Dept. ESAT, Light and Lighting Laboratory, KU Leuven, Technology Campus Ghent, Gebroeders De Smetstraat 1, BE-9000 Ghent

E-mail: nick.rondelez@kuleuven.be 


\section{Introduction}

The last couple of decades, efficiency and low power have become increasingly important, especially for indoor lighting ${ }^{1-5}$. This has caused state-of-the-art LEDs to be the new standard for both home and workplace lighting. These sources have superior efficacy and lifetime, a small footprint and the possibility to be dimmed, when comparing to traditional light sources.

Office buildings account for $40 \%$ of the total energy consumption in the European Union, making it a significant source of greenhouse gasses. Artificial lighting accounts for an important portion of this number ${ }^{6,7}$. This portion could decrease by using LEDs and controlling their light output depending on the amount of incident daylight and occupancy of the room. Such conditions can be monitored by introducing sensors in the luminaire. Moreover, this could also significantly increase lighting comfort by matching the European standard EN 12464-1 for indoor workplace lighting more accurately. There are many papers describing occupancy-based adaptive control systems monitoring the illuminance distribution resulting from the luminaire output and incident daylight, combined. The output of the luminaires is then adjusted in order to achieve a desired target illuminance ${ }^{8-13}$. Such smart lighting clearly has major advantages in terms of efficiency, lighting quality and controllability by the user. In all of these cases, the output of the different light sources is controlled through software. Modern light utilization research is epitomized by innovations in software, rather than inventing hardware to achieve similar or improved functionality.

In the past decade, a substantial amount of effort has gone into the development of LEDs with a small footprint and high luminous flux. Such small étendue light sources allow the design of very compact optical systems for spotlighting or automotive headlights ${ }^{14}$. Major advances in freeform optics design open up lots of new possibilities, where virtually any radiation pattern can be generated using freeform lenses or reflectors ${ }^{15-17}$. Unfortunately, these freeform design methods are based on point sources, which means that LEDs with a relative small footprint are needed. If not, the resulting pattern will deviate strongly from what it is designed for. Although freeform lenses can be used to create complex illumination patterns, these patterns are of course fixed in time. When more complex illumination requirements arise in which the desired radiation pattern has to vary over time, this is not possible.

In 2015, OSRAM Corporate Innovation demonstrated an innovative lighting fixture, of which the resulting radiation pattern could be dynamically adapted ${ }^{18}$. This system made use of 61 narrowbeam LED modules, which can be separately dimmed in order to adapt the emitted light in each direction. A remote control allows to generate varying lighting scenes by manipulating the emitted light flux from each of the different LED modules. This novel system illustrated for the first time that, in addition to control of intensity and colour, control of light direction is also very important for the end-user. According to lighting industry professionals, it is even ranked as more important than CCT and hue ${ }^{18}$. Even though the functionality of the system was quite innovative, the optical design remained trivial. In essence, the radiation pattern is still adjusted by dimming individual LEDs, in which every LED illuminates a specific spatial region. This clearly limits the tailorability of the illumination pattern.

Recently, a lighting system generating different radiation patterns has been designed to account for a changing arrangement of the useable area in a production hall, using reflectors ${ }^{19-21}$. A major disadvantage of using reflectors for spatial tuneable lighting is that these usually have a negative impact on the system size or are unable to capture all emitted light, reducing the maximum achievable efficiency.

Motorized spotlights ${ }^{22}$ are another solution to achieve a changing illumination distribution. These consist of a high-power LED with refractive beam collimation optics, mounted onto an actuator 
with two DC motors driving the two-axis rotation of the spotlight. This approach however implies that the position from which the light is emitted, changes slightly with varying direction. In addition, because the full lighting module is rotated, quite heavy and bulky motors are necessary. Other interesting examples of dynamic beam shaping fixtures are lighting fixtures ${ }^{23,24}$ that allow a dynamical adjustment of the beam divergence by using an electronically controllable diffuser ${ }^{25}$.

In the present paper, the goal is to design a lighting system, which allows a dynamical adjustment of both the beam direction and beam divergence of the emitted light, in a continuous manner. This results in a highly tailorable illumination distribution. Lenses are used to keep the overall system dimensions limited. The amount of electro-mechanical components is also limited and the weight of these components is kept to a minimum. Light is furthermore emitted from a static position, independent of the direction in which the beam is steered.

Section 2 of this paper describes the design of the optical system. A detailed discussion of the design procedure and simulation results is provided. A prototype of the system has been realised, of which the performance is experimentally characterized in Section 3. These experimental results are compared with the simulations results. Finally, a conclusion is drawn in Section 4.

\section{System configuration}

The goal is to design a novel optical configuration that realises a variable illumination pattern by controlling two deflection angles, $\theta$ and $\phi$, and the beam divergence angle $\beta$, as indicated in Figure 1. This system ought to illuminate a room of $4 \mathrm{~m}$ by $4 \mathrm{~m}$ when placed at a height of $3 \mathrm{~m}$. To reach the corners, the beam deflection angles $\theta$ and $\phi$ must be continuously controllable between $+/-22^{\circ}$. The beam divergence $\beta$ needs to be adjustable in order to make the resulting spot size change and provide a minimal illuminance of $250 \mathrm{~lx}$.

For any lighting system, compactness, reliability and efficiency are important features, especially for indoor lighting. Hence, it is important to shape the light with small optical components. The system configuration should furthermore use as little as possible moving components to ensure compactness and robustness. To realise the desired optical functionality, a focus tuneable lens (FTL) is used for controlling the divergence angle $\beta$ and a two-axis rotatable mirror is used to control the deflection angles $\theta$ and $\phi$.

At first glance, one would expect that the optimal system configuration

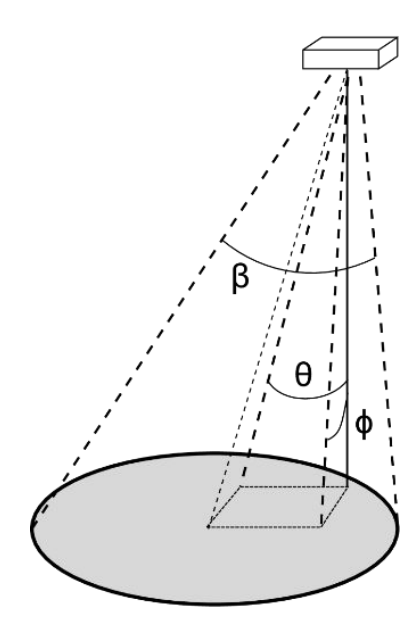

Figure 1 The desired functionality with variable twoaxis beam deflection angles $\theta$ and $\phi$ and beam divergence $\beta$. would consist of an LED with a classic beam collimation optic (e.g. a TIR lens), followed by an FTL and a rotatable mirror. However, this would mean that the light bundle after the FTL towards the mirror is diverging. The configuration therefore demands for a quite large mirror in order to reflect all the emitted light, when considering a relative wide interval for $\theta, \phi$ and $\beta$. This issue can be tackled by using an optical system that focusses the emitted light by the LED towards a specific point after the FTL, in which a compact rotatable mirror can be positioned. Figure 2 illustrates this principal idea. 


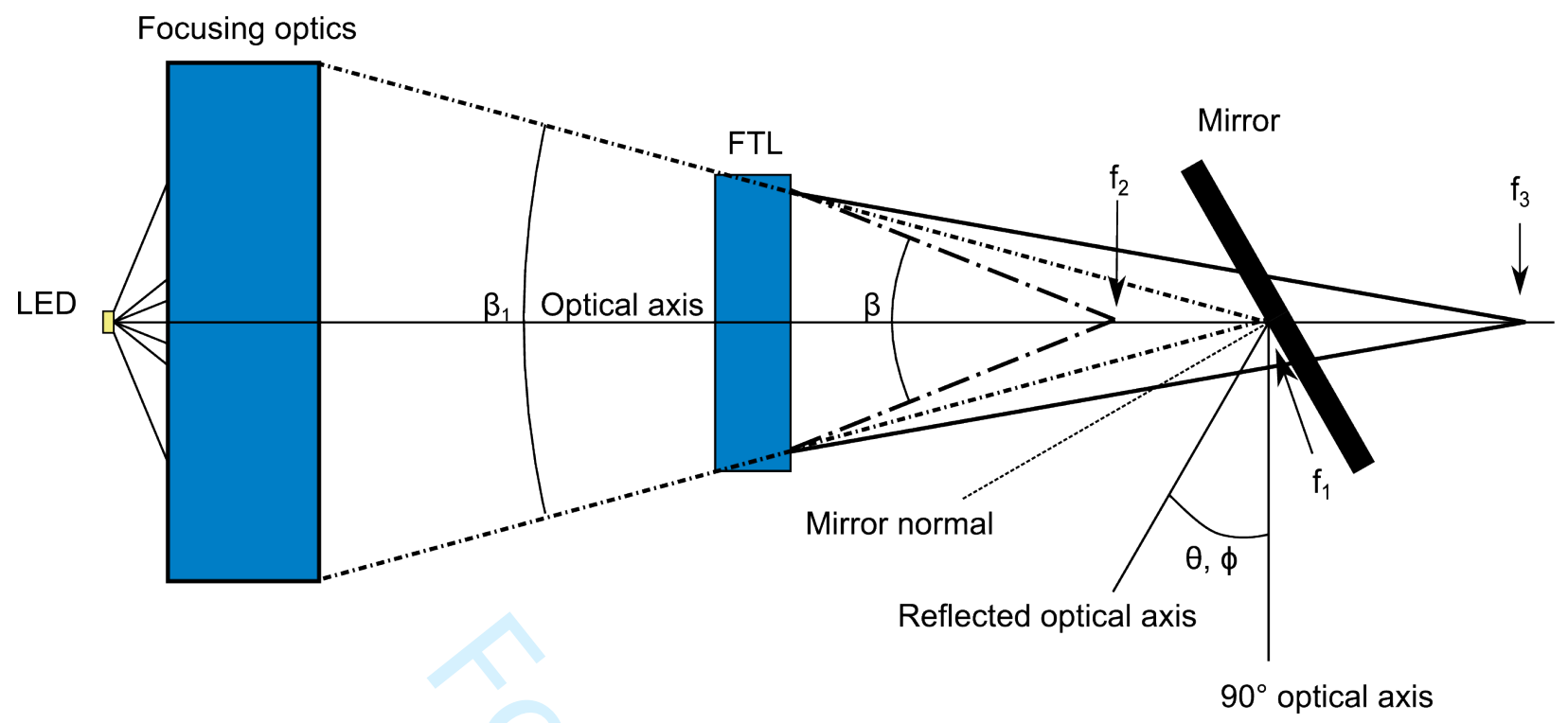

Figure 2 Proposed system: focusing optics direct all emitted light from the LED into the focal point $f_{1}$ with a beam divergence $\beta_{1}$, on a rotatable mirror. An FTL shifts the focal point in front of the mirror $\left(f_{2}\right)$ or behind the mirror $\left(f_{3}\right)$ in a continuous manner, thereby adjusting the beam divergence $\beta$. Tilting the two-axis mirror changes the beam deflection angles $\theta$ and $\phi$. These are the angles between the reflected optical axis and $90^{\circ}$ optical axis. If $\theta=0^{\circ}$ and $\phi=0^{\circ}$, the beam is incident on the area right below the fixture.

The focusing optics that shape the emitted light by the LED towards a focal point consists of two aspherical lenses. To minimise the size of these lenses, the first lens has a flat entrance surface and is positioned directly on top of the LED. This first lens functions as a collimator. The second lens then converges all incident rays towards a point $f_{1}$, which results in a certain beam divergence angle $\beta_{1}$ behind this focal point. In this point, the rotatable mirror is positioned, since the minimal spot size is obtained there, resulting in the smallest possible mirror. The FTL is placed before the mirror such that its aperture is fully illuminated by the incident light beam. This FTL can shift the focal point in front of the mirror $\left(f_{2}\right)$ or behind the mirror $\left(f_{3}\right)$ in a continuous manner, adjusting the beam divergence $\beta$ of the light bundle. The dual axis rotatable mirror can adjust the beam direction as to match the required location by adapting both deflection angles $\theta$ and $\phi$.

For the optical simulations, the ray-tracing software LightTools ${ }^{26}$ is being utilised. This software is widely used for the optical design of illumination systems. It allows users to implement almost arbitrary component shapes, which enables the simulation of complex illumination configurations.

\subsection{Design of the optical system}

The lighting system was designed in such a way that it is able to illuminate a $4 \mathrm{~m} \times 4 \mathrm{~m}$ area by tilting the mirror to the required position. The lighting system is positioned in the centre of this square area at a height of $3 \mathrm{~m}$. Figure 3 shows the final optical configuration. 


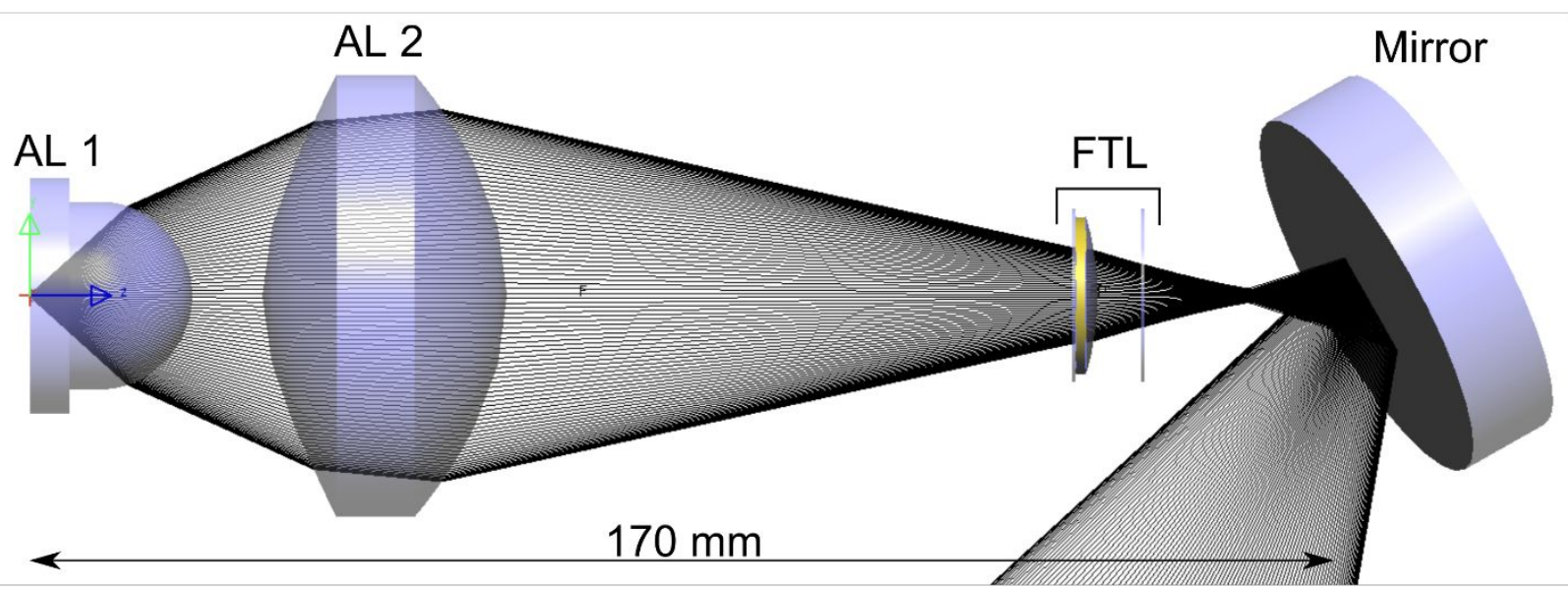

Figure 3 Optical configuration in LightTools using an ideal point source emitting in the full upper hemisphere. The two lenses on the left are aplanatic lenses (AL1 and AL2), focusing the light into a point. The FTL shifts the focal point in front of the mirror, which allows controlling the beam divergence $\beta$. The mirror deflects the beam towards the desired region by adjusting both $\theta$ and $\phi$.

The light source is located in the origin, on the left. While the focusing lenses were designed for a point source, in reality, a compact LED from OSRAM was used, the OSLON® Boost HX LED. This LED has a small footprint (emitting surface of $1.25 \mathrm{~mm} \times 1.59 \mathrm{~mm}$ ), a high lumen output (up to $2120 \mathrm{Im}$ ) and a high luminance of $200 \mathrm{~cd} / \mathrm{mm}^{2}$. The $\mathrm{x}$, $\mathrm{y}$ chromaticity coordinates of the LED are $(0.336,0.364)$. The radiometric power emitted by the LED in the simulations is $1.4 \mathrm{~W}$, corresponding to a photometric flux of $480 \mathrm{Im}$ and $23 \%$ of the maximum output of the LED. This output corresponds with the lumen output used during the experimental characterisation of the system.

As focusing lenses, two aplanatic lenses ( $A L 1$ and $A L 2$ ) were used with generalized Cartesian ovals as lens surfaces, except for the first planar lens surface, which comes directly after the light source. Such generalized Cartesian ovals transform a spherical input wave front into a spherical output wave front, and can therefore be used to image an on-axis object point into a perfect image point without spherical aberration. The two aplanatic lenses were designed with a self-written python script, using a 2D ray mapping approach ${ }^{27}$. The amount of refraction is equally distributed over the different lens surfaces such that chromatic aberration is minimised. Both lens thickness and distance between the lenses have a direct influence on the resulting performance and the overall system size. These parameters were therefore optimized such that a compact focusing system was obtained which performs well for the extended light source that was used in practice. The first lens (AL 1) functions as a collimator lens; the second lens (AL 2) is an aplanatic condenser. The two lenses focus all of the emitted light by the LED towards a focal point with a resulting beam divergence angle $\beta_{1}$ of $30^{\circ}$.

After these lenses, the FTL (Optotune, EL-16-40-TC-VIS-20D ${ }^{28}$ ) is positioned. This FTL consists of a container, filled with an optical fluid and sealed off with an elastic polymer membrane. An electromagnetic actuator exerts pressure on the container, which leads to a deflection of the lens. As a result, the focal length of the FTL is controlled by the positive or negative current that flows through the coil of the actuator, consuming an absolute maximum power of $1 \mathrm{~W}$. The used lens has a clear aperture of $16 \mathrm{~mm}$ and the focal power can be varied from -10 dpt (with maximal negative current) to $10 \mathrm{dpt}$ (with maximal positive current). The FTL can thus shift the focal point in front of the mirror and behind the mirror in order to control the beam divergence of the reflected light. The two larger diameter plates in Figure 3 are the cover plates of the FTL. The behaviour of 
the lens is mimicked in LightTools by calculating the curvature of the simulated FTL to match the theoretical possible focal lengths of the FTL, ranging from $-0.1 \mathrm{~m}$ and $+0.1 \mathrm{~m}$.

Finally, a highly reflective (>97.5\%), silver coated mirror (Thorlabs, PF20-03-P01) is placed in the focal point $\mathrm{f}_{1}$. The mirror has a diameter of $50.8 \mathrm{~mm}$ so that independent of the FTL setting and mirror orientation, all light is incident on the mirror and thus deflected. The mirror is mounted on a dual-axis actuator with the rotation axis in the centre of the mirror. This actuator can control the beam deflection angles $\theta$ and $\phi$ from $-22^{\circ}$ to $22^{\circ}$, which is necessary to reach all corners of the target area. The reason that this mirror is being used and not a micro-electromechanical (MEMS) mirror, is due to the fact that it is not possible to make the beam size small enough such that all light can be deflected. In general, MEMS mirrors are relatively small and thus not directly suitable for this application.

\subsection{Simulated performance}

Although the system was designed for a $4 \mathrm{~m} \times 4 \mathrm{~m}$ target plane, both the target plane dimensions and distance towards the target were reduced with a factor of two during the experimental characterisation for practical convenience. Therefore, the simulated lighting performance at a surface receiver of $2 \mathrm{~m} \times 2 \mathrm{~m}$ located at a distance of $1.5 \mathrm{~m}$ from the centre of the mirror is shown. These results can be easily scaled to other dimensions because the receiver is still located in the far field in this way.

The overall efficiency of the system, defined as the ratio of the radiometric power incident on the receiver to the total emitted radiometric power, is $69 \%$. The main loss factor is the Fresnel losses at the various lens surfaces. By coating the two aplanatic lenses with an anti-reflection coating, a significant gain in the system efficiency could already be obtained.

Below, both the performance for specific tilt angles of the mirror is illustrated, as well as the performance with varying focal power of the FTL. The light beam can be aimed to every position on the receiver by tilting the mirror in the corresponding direction, as illustrated in Figure 4, with the FTL turned off.
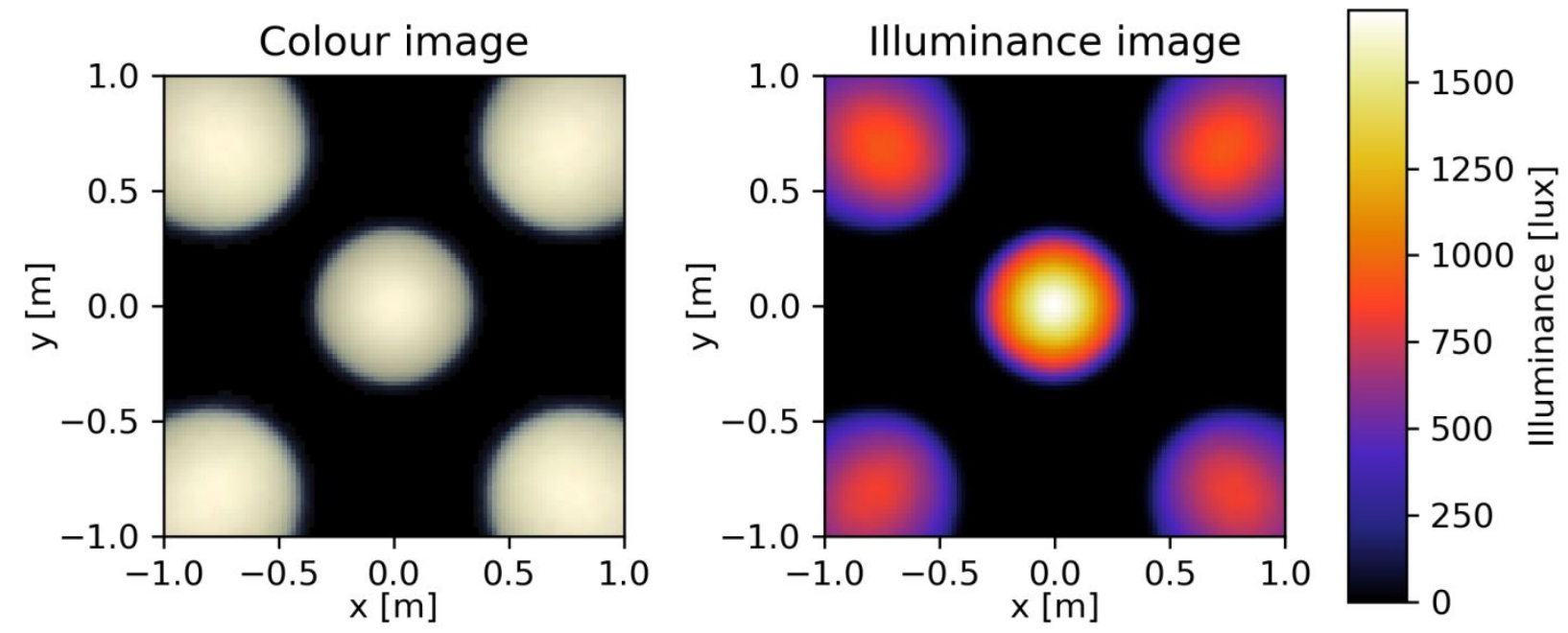

Figure 4 Simulation results on a $2 m \times 2 m$ surface receiver of $101 \times 101$ bins at a distance of $1.5 m$ with the FTL turned off, for five different mirror positions combined. Both graphs show that with the current design, the required spatial 
functionality can be obtained. The colour image (left) shows no visible chromatic aberrations. The illuminance image (right) shows smooth transitions in the distributions.

Both colour and illuminance images are shown for five different mirror positions. The total emitted luminous flux by the LED is $23 \%$ of the maximum output. Analysis of the simulated colour differences shows an average colour difference du'v' $=0.0048$. This implies that there are no significant colour effects caused by the designed lenses and mirror. The total luminous flux for the mirror in the centre position is $332 \mathrm{Im}$ and the peak illuminance value is $1707 \mathrm{Ix}$. For the corner positions, the maximum illuminance decreases due to the larger spreading of the beam, but the total luminous flux is similar. The LED output could be synchronised with the mirror tilt such that the peak illuminance value remains constant for every mirror position.
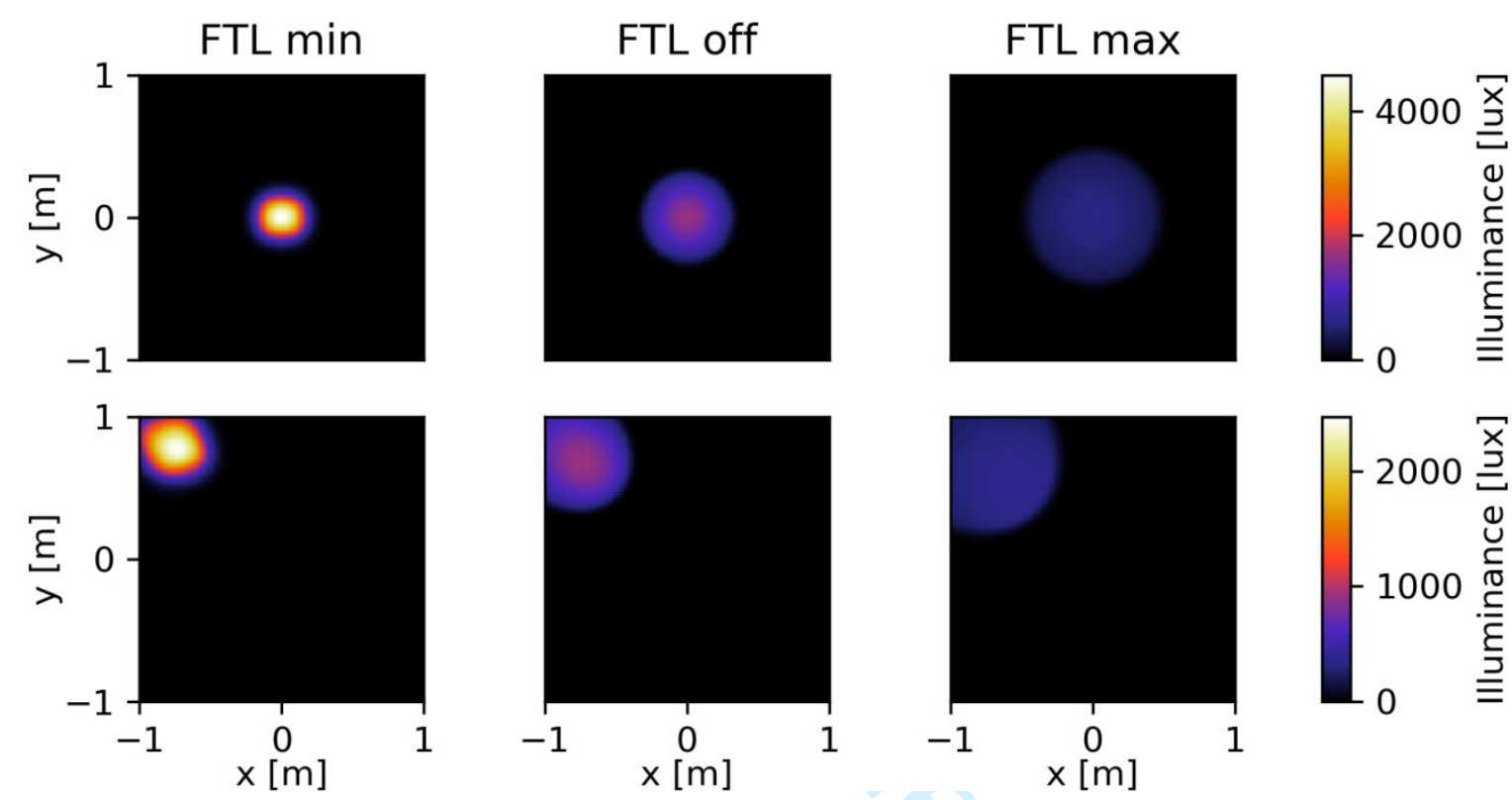

Figure 5 Simulated illuminance pattern for different FTL settings and two mirror positions. The top row shows the evolution of the illumination for different FTL settings with the mirror aimed towards the centre. The bottom row shows the same FTL settings with the mirror aimed to a corner position. FTL min corresponds to a current of -250 mA and focal length of $-0.1 \mathrm{~m}$. With the FTL in the off state, the focal length is infinity. A current of $+250 \mathrm{~mA}$ is used for the FTL max setting, with the focal point located at $+0.1 \mathrm{~m}$.

Figure 5 shows the illuminance distribution when the mirror is aimed towards the centre position (top) and towards the top-left corner position (bottom). The setting of the FTL has a large impact on the illuminance levels for a constant LED output. Spot sizes (defined as $5 \%$ of the centre value) for the top row are $0.44 \mathrm{~m}, 0.68 \mathrm{~m}$ and $1 \mathrm{~m}$ for the min, off and max setting of the FTL respectively. The maximum illuminance is $4581 \mathrm{~lx}, 1707 \mathrm{~lx}$ and $632 \mathrm{~lx}$ from left to right for the top row. Note that these values apply for a receiver of $2 \mathrm{~m} \times 2 \mathrm{~m}$ at a distance of $1.5 \mathrm{~m}$. In the $4 \times 4 \mathrm{~m}$ case, the spot sizes would double and the illuminance levels would decrease by a factor of four. The displayed spot sizes and positions were obtained with the extreme settings for the FTL and mirror, but continuous adjustment of the spot size and position within these boundaries is of course possible.

The designed system can dynamically illuminate an area of $2 \mathrm{~m}$ by $2 \mathrm{~m}$ with a beam of which the size is continuously adjustable between $0.44 \mathrm{~m}$ and $1 \mathrm{~m}$ (for the centre position). The rotatable mirror allows illumination of any position on the target plane by tilting the mirror towards the 
corresponding position. The limits for the beam divergence $\beta$ are calculated from the simulated spot sizes and vary between $16.6^{\circ}$ and $37^{\circ}$. With the FTL turned off, the beam divergence is $29^{\circ}$. The deflection angles $\theta$ and $\phi$ that are necessary to reach the corner positions is $+/-22^{\circ}$.

\section{Experimental characterisation}

\subsection{Measuring setup}

The designed system was realised in practice on an optical breadboard. The final optical system can be seen on the left-hand side of Figure 6. The two aplanatic lenses were CNC-milled from PMMA by an external company. The first lens is directly mounted on top of the heatsink with the high-power LED. The second lens is mounted on the breadboard with a typical lens holder. Next, the Optotune FTL is positioned such that its aperture is fully illuminated by the incident light beam. Finally, the rotatable mirror is positioned with its centre in the focal point of the converging light beam, when the FTL is in the off-state. The actuator that rotates the mirror was developed in such a way that the centre of rotation comes very close to coincide with the centre of the mirror.
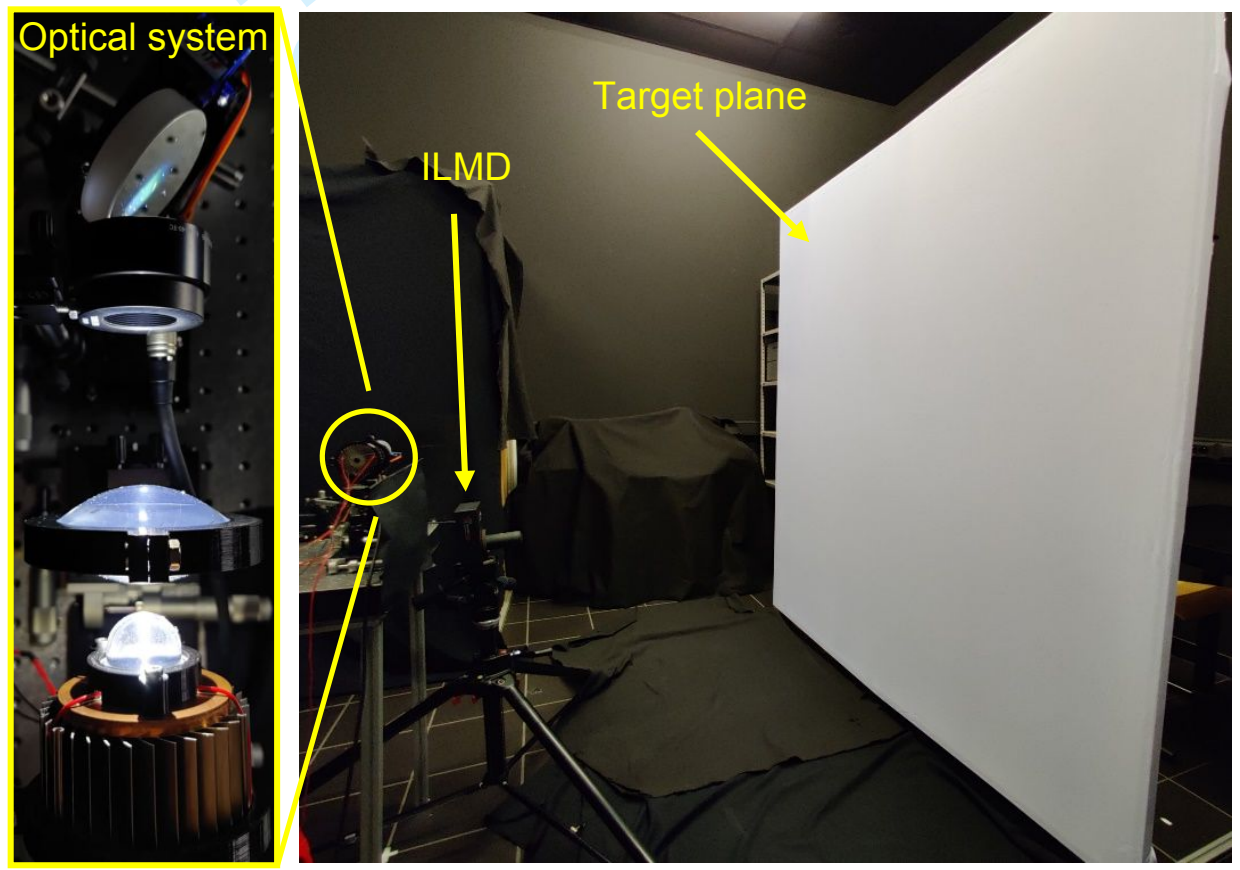

Figure 6 Optical system (left) and measuring setup (right). The left-hand side shows the LED and first aplanatic lens mounted on a heat sink. It is followed by the second aplanatic lens, FTL and rotatable mirror. On the right, the prototype is placed in the centre of the $2 \mathrm{~m} \times 2 \mathrm{~m}$ target plane at a distance of $1.5 \mathrm{~m}$. The imaging luminance measuring device (ILMD) is positioned $0.2 \mathrm{~m}$ below the centre of the lighting system, with its optical axis towards the centre of a white screen that acts as a Lambertian scattering target plane.

To evaluate the tuneable illumination system in terms of deflection angle and beam divergence, the resulting illuminance distribution should be measured and compared with the optical simulations. In order to do so, a white screen of $2 \mathrm{~m} \times 2 \mathrm{~m}$ functions as target plane. The resulting luminance pattern for various settings of the $\mathrm{FTL}$ and rotating mirror was measured with an imaging luminance measuring device (TechnoTeam LMK6-12 colour). This luminance camera was positioned just below the tuneable lighting system, and thus also at a distance of $1.5 \mathrm{~m}$ from the white screen. We verified that the reflected light from the screen followed a Lambertian distribution quite closely. This implies that the illuminance distribution of the incident light at the screen can be directly derived from the measured luminance image following equation (1). 
In this equation, $E$ is the incident illuminance in lux, $L$ is the reflected luminance in $\mathrm{Cd} / \mathrm{m}^{2}$, and $\rho$ is the screen reflectance. This equation assumes that the screen reflectance is independent of both position and wavelength of the incident light (within the visible spectrum). We verified with a dual beam xenon flash spectrophotometer (UltraScan ${ }^{\circledR} \mathrm{PRO}$ ) that both conditions were sufficiently met (deviations below $5 \%$ ) for the used screen.

In addition to luminance images, colour images were also registered with the ILMD. These colour images of the reflected light are also directly related to the colour of the incident light at each position; due to the fact that the reflectance of the used screen is independent of both position and wavelength.

\subsection{Measured performance}

The colour and illuminance distribution was measured for the same spot locations as in the simulations. These five spot locations illustrate that the tuneable lighting system can illuminate any location on the screen.
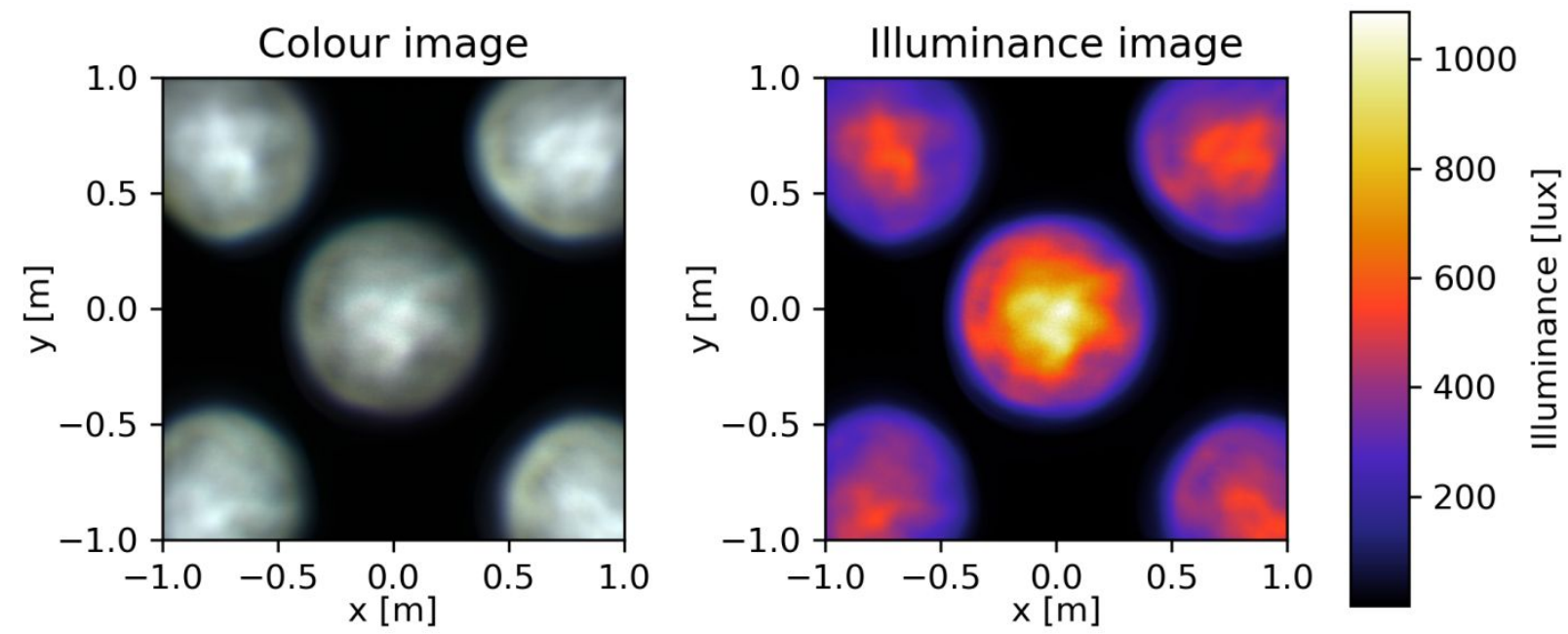

Figure 7 Measurement results on a $2 \mathrm{~m} \times 2 \mathrm{~m}$ receiver at a distance of $1.5 \mathrm{~m}$. The FTL is turned off. On the left, the colour image is generated by combining the measurement results for five different mirror positions. At the edges of the spots, slight colour effects show up. All corners of the room are reached with the setup. The illuminance image shows that the distribution is not uniform.

Figure 7 shows that due to chromatic aberrations, colour effects appear at the edges of the spots. Colour difference analysis on the area with illuminance levels above $5 \%$ of the peak value, result in a du'v' of 0.0735 . Increasing the area to levels above $1 \%$ of the peak value, gives a du'v' $=$ 0.1828 . This strong increase of more than 0.1 indicates that the largest colour differences are present at the borders of the beams, caused by chromatic aberration. A du'v' $=0.0735$ suggests that colour uniformity, when the chromatic aberration at the edges is not taken into account, is still an issue. 

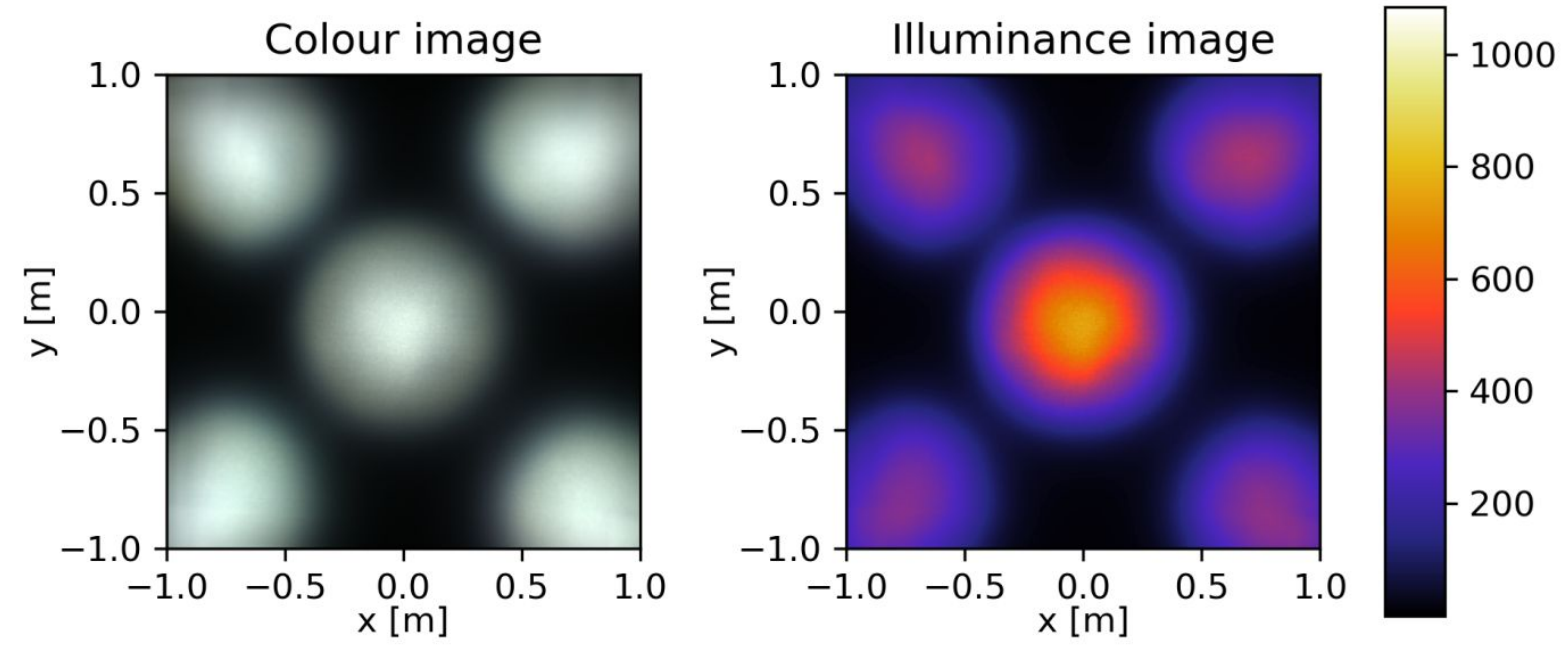

The peak illuminance level is $1084 \mathrm{~lx}$, which is almost $700 \mathrm{~lx}$ lower than the simulated value. This deviation can be fully attributed to the larger beam spreading in the experimental case. This can be concluded from the fact that the measured total luminous flux is $325 \mathrm{Im}$, which is in agreement with the simulated value. Both colour and illuminance uniformity is a problem. Structures are clearly visible in the illuminance pattern, which is unacceptable and cumbersome for illumination applications. The main cause of the uniformity issues and larger beam spreading is directly related with the quality of the custom-made PMMA lenses. Deviations of the surface topology of the fabricated lenses compared with the designed lenses result in discontinuities in the illumination pattern and less focusing of the light. This was immediately clear when the resulting light distributions of the LED in combination with the aplanatic lenses alone were evaluated.

However, the non-uniform illumination can be easily improved by introducing a highly efficient surface diffuser after the mirror. This diffuser (C-HE05 from Brightview Technologies) has two functionalities: smoothing the illumination pattern and increasing colour mixing. The resulting colour and illuminance images of the system with this diffuser can be seen in Figure 8.

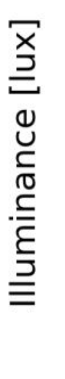

Figure 8: Measurement results on a $2 \mathrm{~m} \times 2 \mathrm{~m}$ receiver at a distance of $1.5 \mathrm{~m}$ with a C-HE05 diffuser placed behind the mirror. The FTL is turned off. All mirror positions are the same as in Figure 7 and the same legend is used. Colour differences are barely visible and irregularities in the pattern smoothed out (left).

Introducing the diffuser slightly decreases efficiency with a factor of $13 \%$ and further broadens the spot diameter. However, the colour and illumination uniformity improves significantly, eliminating the aforementioned uniformity concerns. For $1 \%$ of the peak illuminance value, du'v' decreases to 0.0627 , indicating that the chromatic aberration is no longer an issue. The colour difference in the centre is not cumbersome and is barely noticeable, making it more suitable for illumination applications. The maximum illuminance is $773 \mathrm{Ix}$, about $300 \mathrm{~lx}$ lower than the case without diffuser. This is a direct result from the diffuser, which spreads out the illuminance pattern and slightly reduces efficiency. 

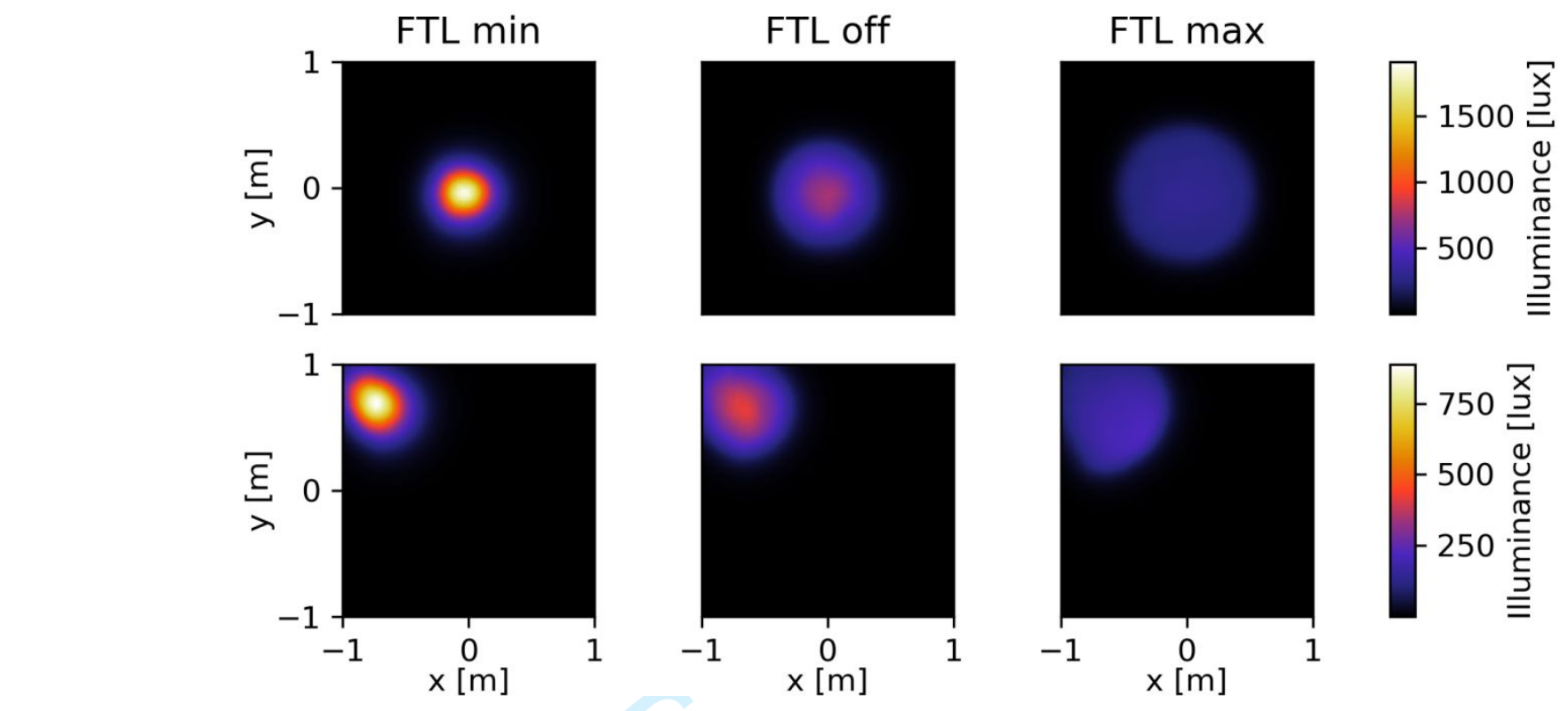

Figure 9 Measured illuminance pattern for different FTL settings and two mirror positions with a C-HE05 diffuser in place. The top row shows the evolution of the illumination when the current through the tuneable lens varies with the mirror aimed at the centre. The bottom row presents the measured illumination distribution for different FTL settings with the mirror aimed to a corner position.

The top row of Figure 9 shows the measured illuminance distributions with diffuser, when the mirror aims towards the centre position, for three different settings of the FTL. The minimum spot diameter is achieved in the FTL min state and equals $0.79 \mathrm{~m}$. In that case, there is a maximum illuminance of $1914 \mathrm{~lx}$. With the FTL in the off state, a maximum illuminance of $773 \mathrm{~lx}$ with a beam diameter of $1.09 \mathrm{~m}$ is obtained. Subsequently, the maximum current setting results in a spot diameter of $1.40 \mathrm{~m}$ and a peak illuminance of $330 \mathrm{~lx}$. The bottom row shows the illumination distributions with the mirror aimed towards the top left corner. Efficiency is experimentally determined to be around $60 \%$.

\subsection{Discussion}

The experimental study shows that the designed configuration can indeed realise the prescribed adaptive functionality. Both beam diameter and direction are adjustable within the desired range. However, there are some rather large quantitative differences between simulations and measurements. The experimentally obtained light distributions show disturbing colour and illuminance non-uniformities in the obtained beam spots. These differences can be attributed to imperfections of the CNC-milled lenses. In our experimental study, these issues have been solved by using a diffuser after the mirror.

Table 1 gives a short summary of the main parameters characterising the simulated and measured system performance. The manufactured aplanatic lenses generate a beam divergence of $34.7^{\circ}$, instead of the $30^{\circ}$ for which they were designed. Combined with the diffuser, the beam divergence increases with another $5^{\circ}$, resulting in a larger spot diameter. The larger spot diameter directly influences the measured illuminance levels.

The slightly lower system efficiency is also caused by introducing the diffuser which has a transmission efficiency of $87 \%$. The use of AR-coated, high quality illumination lenses could certainly improve both the beam shaping performance and efficiency, although it might still be necessary to use an additional diffuser to hide imperfections of the FTL. 


\section{Conclusion}

The designed optical system fulfils the targeted requirements for the tuneable beam size and beam direction. Compared to the simulated performance, a larger beam divergence is obtained in practice. This influences both the obtained illuminance levels and spot sizes. Manufacturing the illumination lenses by a more refined technology could result in a prototype behaviour that comes closer to the simulated performance.

We remark that a spot lighting fixture with a tuneable beam size and beam direction could also be obtained by positioning a lighting fixture with a tuneable lens or diffuser on a motorized rotation stage. However, the approach that is presented in this paper has two major advantages. With a moving head light fixture, the position from which the light is emitted changes with direction. The present solution on the other hand, has a fixed emission point, namely the centre of the used mirror. This enables e.g. that the reflected light beam by the mirror shines through a fixed aperture, in order to hide the lighting system behind a wall, ceiling or mechanical housing. The current design still uses an electro-mechanical component. However, the size and weight of this component are very limited when compared to mounting a full lighting fixture on a rotation stage.

Applications for the presented lighting fixture could range from adaptive stage- and museum lighting, to general lighting applications with a tuneable radiation pattern. By adding a camera to the system, both the location of a person as well as the illumination around that person could be determined. The beam size, beam direction and light output of the fixture could then be adjusted as is required. For more static applications, e.g. the illumination of art objects, the tuneable beam size and direction could be used to assure optimal lighting conditions in various circumstances.

\section{Declaration of conflicting interests}

The authors declare no potential conflicts of interest with respect to the research, authorship and/or publication of this paper

\section{Funding}

Nick Rondelez received financial support for this research from the Technology Campus Ghent (KU Leuven) through BAM doctoral funding.

\section{ORCID iD}

Nick Rondelez https://orcid.org/0000-0001-8356-9988 


\section{References}

1. Khan N, Abas N. Comparative study of energy saving light sources. Renewable and Sustainable Energy Reviews 2011; 15: 296-309.

2. Jacob B. Lamps for improving the energy efficiency of domestic lighting. Lighting Research and Technology 2009; 41: 219-228.

3. Tan ST, Sun XW, Demir H V., Denbaars SP. Advances in the LED materials and architectures for energy-saving solid-state lighting toward lighting revolution. IEEE Photonics Journal 2012; 4: 613-619.

4. Duggal AR, Heller CM, Shiang JJ, Liu J, Lewis LN. Solution-processed organic lightemitting diodes for lighting. IEEE/OSA Journal of Display Technology 2007; 3: 184-192.

5. Gayral B. LEDs for lighting: Basic physics and prospects for energy savings. Comptes Rendus Physique 2017; 18: 453-461.

6. European Parliament and Council. Directive 2010/31/EU of the European Parliament and ofthe Council of 19 May 2010 on the energy performance of buildings. Official Journal of the European Union 2010; L 153/13-35.

7. European Parliament and Council. Directive 2018/844/EU of the European Parliament and of the Council of 30 May 2018 amending Directive 2010/31/EU on the energy performance of buildings and Directive 2012/27/EU on energy efficiency. Official Journal of the European Union 2018; L158/75-91.

8. Mandal P, Roy B. Adaptive dimming scheme based daylight pipe integrated indoor lighting system under Perez All-weather sky model, 2016 IEEE International WIE Conference on Electrical and Computer Engineering (WIECON-ECE). Pune, India: IEEE, 19 December 2016: pp. 221-224.

9. Gilani S, O'Brien W. A preliminary study of occupants' use of manual lighting controls in private offices: A case study. Energy and Buildings 2018; 159: 572-586.

10. Mahajan SA, Markande SD. Design of intelligent system for indoor lighting, 2016 International Conference on Computing Communication Control and automation (ICCUBEA). Pune, India: IEEE, 12 August 2016: pp. 1-4.

11. Matta S, Mahmud SM. An intelligent light control system for power saving, IECON 2010 36th Annual Conference on IEEE Industrial Electronics Society. Glendale, AZ, USA 7 November 2010: pp. 3316-3321.

12. Caicedo D, Pandharipande A, Willems FMJ. Daylight-adaptive lighting control using light sensor calibration prior-information. Energy and Buildings 2014; 73: 105-114.

13. Pan M-SS, Yeh L-WW, Chen Y-AA, Lin Y-HH, Tseng Y-CC. Design and Implementation of a WSN-Based Intelligent Light Control System, The 28th International Conference on Distributed Computing Systems Workshops. Beijing, China: IEEE, 17 June 2008: pp. 321326.

14. Wall F, Martin PS, Harbers G. High-power LED package requirements, Third International Conference on Solid State Lighting. San Diego, CA, USA: SPIE, 26 January 2004: pp. 8592. 
15. Desnijder K, Hanselaer $P$, Meuret $Y$. Flexible design method for freeform lenses with an arbitrary lens contour. Optics Letters 2017; 42: 5238-5241.

16. Desnijder K, Deketelaere W, Ryckaert W, Hanselaer P, Meuret Y. Efficient Design Method of Segmented Lenses for Lighting Applications with Prescribed Intensity and Low Peak Luminance. LEUKOS - Journal of Illuminating Engineering Society of North America 2019; 15: 281-292.

17. Bruneton A, Bäuerle A, Wester R, Stollenwerk J, Loosen P. High resolution irradiance tailoring using multiple freeform surfaces. Optics Express 2013; 21: 10563.

18. Pereyra RM, Quilici MA, Ryu S-C, Sarkisian AR. 11-2: Invited Paper: Spatial and Beam Control in Solid State Lighting Applications, SID Symposium Digest of Technical Papers. San Francisco, CA: Wiley, 22 May 2016: pp. 114-117.

19. Leśko M, Różowicz A, Baran K, Wachta H, Lesko M, Wachta H, Baran K, Rozowicz A. A Concept of an Adaptive Luminaire with Variable Luminous Intensity Distribution, 7th Lighting Conference of the Visegrad Countries (LUMEN V4). Trebic, Czech Republic: IEEE, 5 November 2018: pp. 1-4.

20. Leśko M, Różowicz A, Baran $\mathrm{K}$, Wachta $\mathrm{H}$. A luminaire with variable light distribution, E3S Web of Conferences. 13 August 2018: pp. 1-9.

21. Leśko $\mathrm{M}$, Różowicz $\mathrm{A}$, Baran $\mathrm{K}$, Wachta $\mathrm{H}$. Adaptive Luminaire with Variable Luminous Intensity Distribution. Energies 2020; 13: 721.

22. Forma Lighting Moto-Ola, Retrieved 27 February 2020 from https://www.formalighting.com/products/indoor-lighting/motorized-remote-controllerlighting/moto-ola.

23. Dynamic Beam Shaper | Targetti Sankey Lighting, Retrieved 27 February 2020 from http://www.targetti.com/en/dynamic-beam-shaper.

24. THE ARCANE - Eulum Variable beam angle and light intensity, Retrieved 28 February 2020 from https://eulum.com/product/arcane-variable-beam-angle/.

25. Lensvector | Digitally Managing Light, Retrieved 28 February 2020 from https://www.lensvector.com/.

26. LightTools 8.7.0 Illumination Design Software, Retrieved 28 February 2020 from https://www.synopsys.com/optical-solutions/lighttools.html.

27. Winston R, Miñano JC, Benitez P, Shatz N, Bortz JC. Nonimaging optics. Cambridge, MA, USA: Elsevier Academic Press, 2005.

28. Optotune - EL-16-40-TC lens, Retrieved 28 February 2020 from https://www.optotune.com/el-16-40-tc-lens. 
Table 1 Quantitative comparison of the simulated system performance compared to the measured performance when a diffuser is in place. The parameters are determined for a $2 m \times 2 m$ target plane.

\begin{tabular}{|l|l|l|}
\hline Parameter & Simulation & Measurement \\
\hline Efficiency & $69 \%$ & $60 \%$ \\
\hline Colour difference du'v' & 0.0085 & 0.0627 \\
\hline Min. spot diameter & $0.44 \mathrm{~m}$ & $0.79 \mathrm{~m}$ \\
\hline Max. spot diameter & $1.01 \mathrm{~m}$ & $1.40 \mathrm{~m}$ \\
\hline Min. illuminance & $632 \mathrm{Ix}$ & $330 \mathrm{Ix}$ \\
\hline Max. illuminance & $4581 \mathrm{Ix}$ & $1914 \mathrm{Ix}$ \\
\hline Beam deflection angle $\theta$ and $\phi$ & $+/-22^{\circ}$ & $+/-22^{\circ}$ \\
\hline Min. beam divergence & $16.6^{\circ}$ & $29^{\circ}$ \\
\hline Max. beam divergence & $37.3^{\circ}$ & $50^{\circ}$ \\
\hline
\end{tabular}


Figure 1 The desired functionality with variable two-axis beam deflection angles $\theta$ and $\phi$ and beam divergence $\beta$.

Figure 2 Proposed system: focusing optics direct all emitted light from the LED into the focal point $f_{1}$ with a beam divergence $\beta_{1}$, on a rotatable mirror. A focus tuneable lens (FTL) can shift the focal point in front of the mirror $\left(f_{2}\right)$ or behind the mirror $\left(f_{3}\right)$ in a continuous manner, thereby adjusting the beam divergence $\beta$. Tilting the two-axis mirror changes the beam deflection angles $\theta$ and $\phi$. These are the angles between the reflected optical axis and $90^{\circ}$ optical axis. If $\theta=0$ and $\phi=0$, the beam is incident on the area right below the fixture. ...4

Figure 3 Optical configuration in LightTools using an ideal point source emitting in the full upper hemisphere. The two lenses on the left are aplanatic lenses (AL1 and AL2), focusing the light into a point. The FTL shifts the focal point in front of the mirror, which allows controlling the beam divergence $\beta$. The mirror deflects the beam towards the desired region. ...5

Figure 4 Simulation results on a $2 \mathrm{~m} \times 2 \mathrm{~m}$ surface receiver of $101 \times 101$ bins at a distance of 1.5 $\mathrm{m}$ with the FTL turned off, for five different mirror positions combined. Both graphs show that with the current design, the required spatial functionality can be obtained in theory. The colour image (left) shows no visible chromatic aberrations. .7

Figure 5 Simulated illuminance pattern for different FTL settings and two mirror positions. The top row shows the evolution of the illumination for different FTL settings with the mirror aimed towards the centre. The bottom row shows the same FTL settings with the mirror aimed to a corner position. FTL min corresponds to a current of $-250 \mathrm{~mA}$ and focal length of $-0.1 \mathrm{~m}$. With the FTL in the off state, the focal length is infinity. A current of $+250 \mathrm{~mA}$ is used for the FTL max setting, with the focal point located at $+0.1 \mathrm{~m}$. ..8

Figure 6 Optical system (left) and measuring setup (right). The left side shows the LED and first aplanatic lens mounted on a heat sink. It is followed by the second aplanatic lens, FTL and rotatable mirror. On the right, the prototype is placed in the centre of the $2 \mathrm{~m} \times 2 \mathrm{~m}$ target plane at a distance of $1.5 \mathrm{~m}$. The imaging luminance measuring device (ILMD) is positioned $0.2 \mathrm{~m}$ below the centre of the lighting system, with its optical axis towards the centre of a white screen that acts as target plane.

Figure 7 Measurement results on a $2 \mathrm{~m} \times 2 \mathrm{~m}$ receiver at a distance of $1.5 \mathrm{~m}$. The FTL is turned off. On the left, the colour image is generated by combining the measurement results for five different mirror positions. At the edges of the spots, slight colour effects show up. All corners of the room are reached with the setup. The illuminance image shows that the distribution is not uniform.

Figure 8: Measurement results on a $2 \mathrm{~m} \times 2 \mathrm{~m}$ receiver at a distance of $1.5 \mathrm{~m}$ with a C-HE05 diffuser placed behind the mirror. The FTL is turned off. All mirror positions are the same as in Figure 7. Colour differences are barely visible and irregularities in the pattern smoothed out (left). The Illuminance image (right) uses the same legend as in Figure 7. .11

Figure 9 Measured illuminance pattern for different FTL settings and two mirror positions with a C-HE05 diffuser in place. The top row shows the evolution of the illumination when the current through the tuneable lens is varied with the mirror aimed at the centre. The bottom row presents the measured illumination distribution for different FTL settings with the mirror aimed to a corner position. 12 